\title{
*Sternum Puncture in Leprosy - a Study of Fifty Cases
}

J. Lowe and Dharmendra.

Leprosy appears to be largely an infection of the reticuloendothelial elements in the body. In the skin and in the nerves infection appears to be of this nature. In "skin" leprosy the endothelial cells lining the blood vessels are frequently affected. The reticulum of the lymphatic glands is frequently affected. Lesions of the internal organs are not uncommonly seen and it is noticeable that the Küpffer cells

*Reprinted from "Leprosy in India," October, 1937, Vol. IX, No. 4. 
of the liver are frequently affected and also the reticulum cells of the spleen.

That the cells in leprous tissue are of the nature of histiocytes has been (lemonstrated by the injection of aniline (lye which is taken up by the cells. Similar findlings have been macle in rat leprosy.

An important part of the reticulo-endothelial system of the body is the bone marrow. Very few references are found in the literature of leprosy to affections of the bone marrow. According to Klingmuller the infection of bone marrow was first observed by Babes and later by other workers but the work on the subject is very meagre. Gass (1936) examined the bone marrow of bones removed from cases of leprosy at operation and found lepra bacilli present almost invariably in cases of cutaneous leprosy.

It appears possible that the bone marrow might be an important focus of leprous infection. In cases of cutaneous leprosy the infection is a widespread one in the various tissues and organs of the body and it is noticeable that in such cases, improvement with disappearance of the bacilli from the skin is frequently followe(l by relapse. It is possible that there may be in the body foci of persistent latent infection one of which might be the bone marrow and that from these foci bacilli are liberated causing relapse. In cases of nerve leprosy the infection is apparently localized in certain tissues of the body. Clinical observation of such cases of leprosy with alternating periods of quiescence and activity suggests that even in cases of nerve leprosy there may be in the body some focus of infection from which bacilli are liberated from time to time. This focus might possibly be the bone marrow.

It therefore seemed desirable that more information should be collected regarding the infection of bone marrow in leprosy and in the various types and phases of leprosy. Our first attempts at investigation of this matter were made at autopsy but antopsy material is very limited in this centre. Nevertheless by trephining the tiba in a few cases of leprosy at autospy we were able to demonstrate that infection of bone marrow is present and is surprisingly heavy in cases of cutaneous leprosy. We were unable to get cases of nerve leprosy to studly by this method, and therefore we sought for means of examining the bone marrow during life.

The only method available seems to be sternal puncture which is being increasingly used for a study of bone marrow in connection with blood diseases. The sternal puncture apparatus consists of a fine trocar and cannula rather like a 
lumbar puncture needle with a special adjustable guard which can be fixed, by means of the screw, at the desired distance from the end. The cannula is made to fit on to the end of a record syringe. The method of obtaining sternal puncture material is as follow's :-

The site for the puncture is the micllle of the sternal opposite the third intercostal space. The patient lies on his back and the skin, subcutaneous tissues and periosteum, at the site of puncture, are anæsthetised with 2 per cent. novacain solution, and about 15 minutes later the puncture is made. The guard is fixed between ${ }_{2}^{1}$ and $1 \mathrm{~cm}$. from the point of the needle, the distance varying according to the thickness of the tissues covering the sternum. The needle is driven straight down with a rotating motion and one can feel the onter bony plate of the sternum being pierced. When the needle has pierced the sternum its further progress is prevented by the guard and it is found that the needle is firmly fixed in the bone. If this is not so, it is probably because the point of the needle is not deep enough and the guard is then moved slightly further up the needle and the needle is inserted a little farther. At this point the trocar is removed and the syringe is attached to the needle and by steady suction about one cubic centimetre of sternal fluicl is withdrawn. The actual piercing of the sternum is not painful with proper anesthesia but a little pain is felt when the Aluid is withdrawn.

\section{Materials cramined.}

Sternal puncture material was taken by this method from 50 patients. They were classified as follows : 32 cases of cutaneous leprosy and 18 cases of nerve leprosy. Since the finding of a few bacilli in the sternal puncture material might possibly be due to the puncture being made through leprous skin, slit smears were made from the skin at the site of the puncture and examined in the ordinary way.

\section{Examinations made.}

The sternal puncture fluids were treated as follows :-

(1) A thick smear was made on a slide, dried, dehæmoglobinised with 3 per cent. acetic acid and stained for acicl-fast bacilli.

(2) The remaining fluid was treated as follows: It was placed in a sterile tube containing alout 0.5 c.c. of 3 per cent. sodium citrate solution. This was centrifuged. The supernatant fluid was removed from the cells and about 4 c.c. of 3 per cent. acetic 
acid was added. After thorough mixing, the tube was again centrifuged, the supernatant fluid being removed and smears made from the deposit.

\section{Findings.}

Of 32 cases of cutaneous leprosy, bacilli were found in the sternal fluid in 16 . Of the 16 cutaneous cases, bacilli were present in the skin of various parts of the body and in the skin covering the sternum of 15 , but in most of the cases the number of bacilli found in the sternal fluid was sufficiently great to make it unlikely that the positive findings were attributable entirely to the needle being infected by passing through leprous skin. We interpret these findings as indicating that the bone marrow of the sternum showed bacilli in approximately 50 per cent. of $\mathrm{C}$ cases examined. On the other hand in 18 cases of nerve leprosy, bacilli were found in the bone marrow in only one case. This case, however, is striking, the number of bacilli found in the bone marrow being considerable whereas no bacilli had been found in the skin of the body.

\section{Discussion.}

These findings show that when bacilli are present in the skin they are often but perhaps not always present in the bone marrow. The failure to demonstrate bacilli by a single puncture of one bone does not rule out the possibility of infection of the bone marrow in other sites. In our experience bacilli are present in the bone marrow in most cases of severe cutarieous leprosy and it would be interesting to find out whether, in cases in which the infection dies out from the skin, the infection still persists in the bone marrow. If so, this may help to explain many cases of relapse. The same remark applies in a modified degree to cases of nerve leprosy. The finding of bacilli in the bone marrow in one of 18 cases cases of leprosy by a single puncture of one bone suggests that a more thorough investigation might demonstrate the presence of bacilli in the bone marrow of a larger number of cases of nerve leprosy. We publish this note largely with a view to stimulating others, who may have facilities for the work, to make a closer study of the bone marrow in leprosy than we have been able to do. A careful study of material taken from post-mortem cases of leprosy of various types might give interesting results.

\section{REFERENCE.}

Gass, H. H. (1937), “Leprosy in India,” Vol. IX, p. 14. 